\title{
Supplementation with Ziziphus Jujuba Suppresses Apoptosis Signals in Neutrophils after Acute Exercise
}

\author{
Seyed Morteza Tayebi', Karsten Krüger², Farahnaz Ebrahimi ${ }^{3}$, Abbas Izadi ${ }^{3}$, Maryam Roushan ${ }^{3}$, Anna V. Nenasheva ${ }^{4}$
}

Affiliations: 'Allameh Tabataba'i University, Faculty of Sport Science, Department of Exercise Physiology, Tehran, Iran, ${ }^{2} J$ ustus-Liebig-Universität Gießen, Department of Exercise Physiology and Sports Therapy, Gießen, Germany, ${ }^{3}$ Islamic Azad University, Kerman Branch, Faculty of Humanity, Department of Exercise Physiology, Kerman, Iran, ${ }^{4}$ South Ural State University (National Research University), Institute of Sport, Tourism and Service, Athletic Performance Enhancement Department, Chelyabinsk, Russia

Correspondence: S. M. Tayebi, Allameh Tabataba'i University, Faculty of Sports Sciences, Department of Exercise Physiology, Shahid Hemmat West, Dehkadeh-y-Olympic, P.O. Box: 1489684511, Tehran, Iran. E-mail: tayebism@gmail.com

\begin{abstract}
It is suggested that jujube might benefit exercise-induced immune fluctuations, specifically on neutrophils' apoptosis regulation, but its cellular mechanism is unclear. This study aimed to investigate the effect of one-week supplementation with Ziziphus jujuba on pro- and anti-apoptotic protein levels of neutrophils in response to a session of circuit resistance exercise. Fourteen young, healthy male students completed a session of circuit resistance exercise (75\% 1RM, nine exercises, three sets) in two groups $(n=7)$. While one group received a placebo, the other group was supplemented with jujube $(0.5 \mathrm{~g} / \mathrm{kg}$ body weight suspended in $2.5 \mathrm{cc}$ distilled water) started a weekday before the exercise session. Blood samples were collected 30 minutes before, immediately, and two hours after the exercise. Neutrophils were collected and pro- (Calpain-1, Bax, Caspase-3) and anti-apoptotic (Calpastatin, XIAP) proteins measured with ELISA. In-

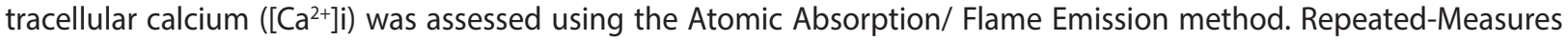
ANOVA was used for the interaction effects of TIMEXGROUP $(3 \times 2)$ at the significance level $(p)$ of 0.05 . The SPSS software was used for analyses. Levels of $\left(\left[\mathrm{Ca}^{2+}\right] \mathrm{i}\right)$, expression of calpain-, and caspase- 3 were increased in response to circuit resistance exercise $(p<0.05)$. In contrast, supplementation with jujube suppressed these changes $(p<0.01)$. The data indicate that a single session of intensive circuit resistance exercise elevated apoptosis signalling in human neutrophils with the involvement of $\left[\mathrm{Ca}^{2+}\right]$ i-Calpastatin-Calpain axis upstream caspase-3. Supplementation with the jujube solution attenuated cell death signalling, possibly by providing energy for neutrophils. Otherwise, the improvement of the antioxidant status might be protective against ROS-induced apoptosis during exercise.
\end{abstract}

Keywords: exercise, resistance exercise, ziziphus jujuba, neutrophil cell, cell apoptosis, intracellular calcium

@MJSSMontenegro

ZIZIPHUS JUJUBA SUPPRESSES NEUTROPHILS' APOPTOSIS AFTER EXERCISE

$\mathrm{http}: / / \mathrm{mjssm} \cdot \mathrm{me} /$ ssekcija $=$ article\&artid $=220$

Cite this article: Tayebi, S.M., Krüger, K., Ebrahimi, F., Izadi, A., Roushan, M., \& Nenasheva, A.V. (2021). Supplementation with Ziziphus Jujuba Suppresses Apoptosis Signals in Neutrophils after Acute Exercise. Montenegrin Journal of Sports Science and Medicine, 10(2), 31-39. doi: 10.26773/mjssm.210905

\section{Introduction}

Neutrophils are an essential part of the innate immune system (Ermert et al., 2013) that are the first responders during the beginning of inflammation, mainly as a result of bacterial infection, environmental stress (Jacobs et al., 2010), and exercise (Kruger \& Mooren, 2014), which are mobilized and migrate towards the sites of inflammation. A single bout of high-intensity exercise causes a marked increase in blood 
leukocyte counts, mainly instigated by the mobilization of neutrophils from the marginal into the circulating pool (Lagranha et al., 2008).

Clinically, exhaustive exercise is followed by a transient impaired immune response called an "open window." During this time, it has been shown that the susceptibility to infections is increased (Lagranha et al., 2005). It is suggested that this phenomenon may result from temporary impaired neutrophil functions and apoptotic cell death (Lagranha et al., 2007). There are conflicting data about the effects of exercise on neutrophil apoptosis. Recently, it has been found that resistance exercise with moderate intensity did not affect neutrophil apoptosis, while intensive resistance exercise, as well as marathon running, decreased the rate of apoptotic neutrophils. Concentric and eccentric treadmill exercise also delayed neutrophil apoptosis during the recovery period (Mooren et al., 2012). In contrast, it has been demonstrated that acute severe exercise-induced an oxidative state in neutrophils resulted in the acceleration of spontaneous neutrophil apoptosis (Syu et al., 2011).

The mechanisms of neutrophil apoptosis during exercise are not completely understood. Previous studies have shown that a single session of exercise induces DNA fragmentation, while mitochondrial membrane depolarization elevates the expression of the pro-apoptotic gene (bax and bcl-xS) and depresses the expression of the anti-apoptotic genes (bcl$\mathrm{xL}$ ) in rat neutrophils (Lagranha et al., 2005; Lagranha et al., 2004). Similarly, increased p53, caspase-3 expression, and p38 MAPK/JNK phosphorylation were shown (Lagranha et al., 2007). However, more recent studies reported beneficial effects of various types of exercise and training on the neutrophils' function and apoptosis in sedentary men (Chen et al., 2018), diabetic patients (Borges et al., 2019) and review of the literature (Chuong et al., 2019); but they did not mention the Bcl-2 family of apoptosis-signalling proteins. A recent study that was modelling the balance between apoptosis and necrosis in neutrophils, but not in exercise and training (Presbitero et al., 2019) explains some mechanism of neutrophils' apoptosis, but there is no data about the Bcl-2 family and calpastatin-calpain-calcium axis.

Regarding anti-apoptotic proteins, not in exercise and training, spontaneous neutrophil apoptosis is known to be regulated by a decrease of calpastatin. Reducing calpastatin levels will cause the constantly active calpains to cleave Bax into an active fragment and deactivate XIAP (Geering \& Simon, 2011). It was also reported that calpastatin and calpain- 1 show critical proximal elements in a cascade of pro-apoptotic events, result in Bax, mitochondria, and caspase- 3 activation. The changed expression affects the life span of Neutrophils under pathologic conditions (Altznauer et al., 2004). To date, no data have been published about the regulation for calpastatin during exercise. As calpastatin and calpain are calcium-dependent proteins, the involvement of the calpastatin-calpain-calcium axis is likely. Therefore, it is an interesting area to be studied.

Several herbal supplements have been tested to blunt the detrimental effects of exercise on the immune system and neutrophil responses. While supplementation of hydrolysed whey protein enriched with glutamine dipeptide before exhaustive treadmill exercise had no effect (Cury-Boaventura et al., 2008), glutamine supplementation prevented exercise effects by reducing neutrophil apoptosis (Lagranha et al., 2005; Lagranha et al., 2007; Lagranha et al., 2004).

Ziziphus jujuba Mill. or the jujube plant, is widely culti- vated, from southwest Europe to China, India, and the Middle East (Gao et al., 2012), especially in Iran (Rodríguez Villanueva \& Rodríguez Villanueva, 2017). It may be considered a functional food, having both nutritional and medicinal uses (Gao et al., 2012). Phytochemical studies showed that jujube fruits have various constituents, such as triterpenic acids (Lee et al., 2003), cerebrosides, flavonoids (Chen et al., 2013), nucleosides (Guo et al., 2013), phenolic acids (Du et al., 2013; Wang et al., 2011), sugars (Li et al., 2013), and amino acids (Guo et al., 2013).

Several studies have confirmed that jujube displays many critical biological properties, including anti-oxidative, neuroprotective, anticarcinogenic, anti-inflammatory, immunomodulatory, cardio-, hepato-, and gastrointestinal protective activities, thus supporting the putative health benefits deriving from its consumption (Plastina, 2016). Accordingly, the jujube might be beneficial for exercise and its acute immunologic consequences, such as neutrophil apoptosis. In previous studies, we demonstrated that one-week supplementation of jujube solution delayed neutrophil apoptosis-induced by an intensive circuit resistance exercise (Tayebi et al., 2014). However, the underlying mechanisms remain unknown.

The current study aimed to investigate the effect of one week of supplementation with jujube solution before a single session of intensive circuit resistance exercise on protein expression of caspase-3, calpastatin, calpain-1, Bax, XIAP, and $\left[\mathrm{Ca}^{2+}\right]_{\mathrm{i}}$ in human neutrophils.

\section{Methods}

\section{Participants}

The present study was approved by the Research Ethics Committee of the Tarbiat Modares University of Medical Sciences and was conducted by the policy statement of the Declaration of the Iranian Ministry of Health that is following the 1964 Helsinki Declaration and its later amendments or comparable ethical standards. Written informed consent was obtained from 14 healthy and young male students. All subjects were requested to complete a medical examination to ensure that they had not taken any regular medication, smoked, consumed alcohol, or taken any regular exercise in the past two months. They also had to state that they are free of cardiovascular or metabolic diseases or recent upper respiratory tract infection symptoms in the month before the start of these tests. The volunteers were randomly assigned to two groups $(n=7)$, including a Circuit Resistance Exercise group with placebo (age: $25 \pm 3$ years, height: $171 \pm 2 \mathrm{~cm}$, weight: $67.5 \pm 4.9 \mathrm{~kg}$ ) and a Circuit Resistance Exercise group ( $\mathrm{n}=7)$ with jujube solution (age: $25 \pm 1$ years, height: $180 \pm 4 \mathrm{~cm}$, weight: $74.1 \pm 5.8 \mathrm{~kg}$ ).

\section{Measurement of exercise capacity}

Participants were weighed three times before the main trial. A strength test was performed at first and second visits to determine one repetition maximum (1-RM) of all participants for each of the nine resistance exercises employed in the study. 1-RM value was determined by adding or removing weights after each attempt. Subjects were permitted to take as much time as they felt necessary for each attempt. A practice session was completed to ensure that each participant could complete the whole exercise session on the third visit and confirm that weight lifting was inducing fatigue by the end of the session. This was confirmed by visual and verbal feedback from participants. Records of 1-RM are presented in Table 1. 
Table 1. Exercise's 1-RM Records of Participants

\begin{tabular}{|c|c|c|c|c|}
\hline Variables (lb) & groups & Median & Minimum & Maximum \\
\hline \multirow{2}{*}{ crunch } & placebo & 134 & 90 & 154 \\
\hline & Jujube solution & 118 & 104 & 171 \\
\hline \multirow{2}{*}{ back extension } & placebo & 155 & 139 & 292 \\
\hline & Jujube solution & 178 & 130 & 660 \\
\hline \multirow{2}{*}{ biceps curl } & placebo & 48 & 38 & 78 \\
\hline & Jujube solution & 65 & 41 & 72 \\
\hline \multirow{2}{*}{ triceps press } & placebo & 51 & 48 & 78 \\
\hline & Jujube solution & 60.5 & 48 & 78 \\
\hline \multirow{2}{*}{ knee extension } & placebo & 152 & 98 & 213 \\
\hline & Jujube solution & 169 & 147 & 218 \\
\hline \multirow{2}{*}{ knee curl } & placebo & 104 & 52 & 128 \\
\hline & Jujube solution & 98 & 60 & 180 \\
\hline \multirow{2}{*}{ standing calf raise } & placebo & 162 & 85 & 211 \\
\hline & Jujube solution & 169.5 & 123 & 199 \\
\hline \multirow{2}{*}{ chest press } & placebo & 69 & 65 & 90 \\
\hline & Jujube solution & 65 & 58 & 105 \\
\hline \multirow{2}{*}{ seated row } & placebo & 124 & 101 & 161 \\
\hline & Jujube solution & 149.5 & 101 & 183 \\
\hline
\end{tabular}

\section{Jujube Preparation}

The semi-dried fruits of Ziziphus Jujube were washed, and seeds were extracted, and the soft parts were removed and were dried at $50^{\circ} \mathrm{C}$ and ground to a powder using a pounder (Vahedi, Fathi Najafi, \& Bozari, 2008).

\section{Combination Assessment of jujube extraction by Gas} Chromatography-Mass Spectrometry (GC-MS)

GC-MS revealed compounds of jujube extraction by semiquantitative methods. The contents of the jujube extraction compounds were quantified using an internal standard

Table 2. Combination Assessment of Jujube Extraction by Gas Chromatography-Mass Spectrometry (GC-MS)

\begin{tabular}{ccc}
\hline Combination & The area under the Peak (\%) & Retention time (min) \\
\hline furfural & 51.33 & 20.21 \\
4-Pyrone & 9.51 & 17.07 \\
Oleic acid & 6.31 & 39.00 \\
Palmic acid & 4.15 & 35.70 \\
Imidazole & 3.03 & 23.59 \\
Cyclononasiloxane & 2.03 & 42.05 \\
Cyclodecasiloxane & 1.75 & 35.63 \\
Oxantin & 1.61 & 10.65 \\
Guanine & 1.58 & 27.20 \\
gamma.-Sitosterol & 1.17 & 44.56 \\
Niphimycin & 1.16 & 26.89 \\
Iron & 1.10 & 45.65 \\
Butanediol & 1.07 & 27.00 \\
Phthalic acid & 1.02 & 45.48 \\
Pentasiloxane & 1.01 & 48.34 \\
Dodecanoic acid & 0.97 & 27.616 \\
octadecamethyl & 0.95 & 32.604 \\
Methyl 2-furoate & 0.92 & 14.28 \\
1,4-dicarbonic acid & 0.86 & 51.997 \\
Tetradecanoic acid & 0.74 & 31.840 \\
Total & 92.27 & \\
\hline
\end{tabular}


(3-octanol, 99\%, Sigma-Aldrich).

Wine hydrophobic compounds were analysed using an Agilent 5975 Mass Spectrometer coupled to an Agilent 7890A Gas Chromatograph (Agilent, Santa Clara, USA). A DB-WAX column $(60 \mathrm{~m} \times 0.25 \mathrm{~mm}$ ID and $0.25 \mu \mathrm{m}$ film thickness) was used for separation. The working factors were as listed: injector temperature of $210^{\circ} \mathrm{C}$, EI source of $230^{\circ} \mathrm{C}$, MS Quad of $150^{\circ} \mathrm{C}$, and transfer line of $210^{\circ} \mathrm{C}$. The primary temperature was $30^{\circ} \mathrm{C}$ for $8 \mathrm{~min}$, which was increased to $150^{\circ} \mathrm{C}$ at a rate of $3^{\circ} \mathrm{C}$ per minute. The injector port temperature was $290^{\circ} \mathrm{C}$, and helium was used as carrier gas at a flow rate of $1.5 \mathrm{ml} / \mathrm{min}$. A total of 15 compounds were positively or tentatively identified by GCMS that contains $92.27 \%$ of the area under the total peak (Table 2).

\section{Exercise Protocol}

Recent studies have reported that appetite hormones, such as ghrelin, increase during fasting and before feeding (breakfast, lunch, and dinner), increasing absorption levels (Tayebi et al., 2012). Accordingly, participants were taken to the test location after a $12 \mathrm{~h}$ overnight fast. All participants performed a session of circuit resistance exercise in two cycles, simultaneously. Each cycle contained nine exercises (Sit Up, back extension, biceps curl, triceps press, knee extension, knee curl, standing calf raise, chest press, seated row, machines were used in all exercises). The test included three non-stop circuits with a 3-minute active rest period between circuits. Each exercise was performed for $30 \mathrm{~s}$ (about 10-14 repeats) with one repeat maximum (1RM) of 75\% (Tayebi et al., 2016; Tayebi et al., 2015). The exercise protocol is shown in Fig.1.

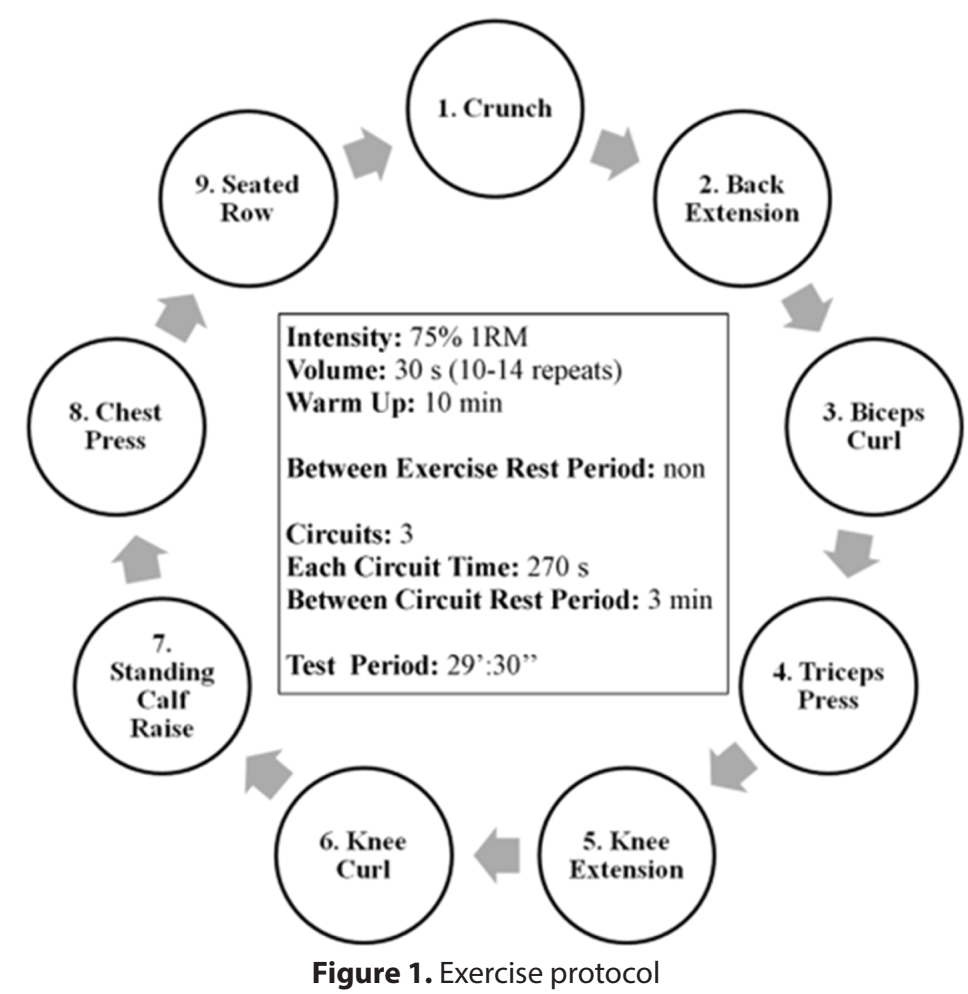

\section{Supplement Protocol and blood collection}

The groups received oral jujube solution $(0.5 \mathrm{~g} / \mathrm{kg}$ body weight in $2.5 \mathrm{cc}$ distilled water) and placebo $(2.5 \mathrm{cc} / \mathrm{kg}$ of body weight in distilled water sweetened by sugar with no calories and coloured by food dye); this supplement was taken each day for a week in a double-blind manner with no physical training within. The groups had a standard diet programme during this week for unification and healthy control.

They received three meals per day: breakfast (10 $\mathrm{kcal} \cdot \mathrm{kg}^{-1} \mathrm{BW}, 70 \%$ carbohydrates, $18 \%$ protein, $12 \%$ fat), lunch (10 kcal. $\mathrm{kg}^{-1}$ BW, 70\% carbohydrates, $18 \%$ protein, $12 \%$ fat) and, dinner ( $18 \mathrm{kcal} \cdot \mathrm{kg}^{-1} \mathrm{BW}, 70 \%$ carbohydrates, $15 \%$ protein, $15 \%$ fat). Subjects in both groups arrived at the test location at 08:00 and rested for about 30 minutes. All subjects performed circuit resistance exercise in two cycles at $08: 30$. On the $8^{\text {th }}$ day and after 12 hours of overnight fasting, the first blood samples were taken at 08:30 AM. The second set of blood samples was taken immediately after exercise at 09:00; subjects then remained seated for 120 minutes. The third set of blood samples was taken at 11:00. The research design and blood collection of the second supplementation protocol is shown in Fig. 2.
Reagents

Anticoagulant ACD (a mixture of citric acid (Sigma Aldrich, Germany), dextrose (Sigma Aldrich, Germany), Dextran 6\% (combination of dextran with at least $100000 \mathrm{MW}$ (grade B, BDH lab, GPR ${ }^{\mathrm{TM}}$, England), $0.6 \mathrm{M} \mathrm{KCl}$ (a mixture of $\mathrm{KCl}$ (Sigma Aldrich, Germany), and dsd $\mathrm{H}_{2} \mathrm{O}$ ), sodium citrate (Sigma Aldrich, Germany), and Deionized Sterile Distilled Water/ds $\mathrm{dH}_{2} \mathrm{O}$ (Baharafshan, B.I.R.D, Iran)), $0.9 \% \mathrm{NaCl}$ (Sigma Aldrich, Germany), and ds $\mathrm{dH}_{2} \mathrm{O}$ ), Phosphate buffered saline/PBS solution (combination of $\mathrm{NaCl}, \mathrm{KCl}, \mathrm{Na}_{2} \mathrm{HPO}_{4}$ (Sigma Aldrich, Germany), glucose (Sigma Aldrich, Germany), $\mathrm{KH}_{2} \mathrm{PO}_{4}$ (Sigma Aldrich, Germany), and $\mathrm{dsdH}_{2} \mathrm{O}$ ) with pH 7.4 and Autoclaved, Hank's Balanced Salt Solution/HBSS without $\mathrm{Ca} \& \mathrm{Mg}\left(\mathrm{NaCl}, \mathrm{KCl}, \mathrm{Na}_{2} \mathrm{HPO}_{4}, \mathrm{KH}_{2} \mathrm{PO}_{4}\right.$, $\mathrm{NaHCO}_{3}$ (Sigma Aldrich, Germany), and $\mathrm{dsdH}_{2} \mathrm{O}$ ) with pH 7.4 and Autoclaved, Ficoll-Hypaque 10.77 (Baharafshan, B.I.R.D, Iran).

\section{Neutrophil isolation}

Neutrophils were purified from venous blood treated with ACD from healthy volunteers by three steps: Dextran sedimentation, Hypotonic lysis, and Ficoll sedimentation (Heit, 2001). Briefly, a solution of $6 \%$ Dextran \& $0.9 \% \mathrm{NaCl}$ 


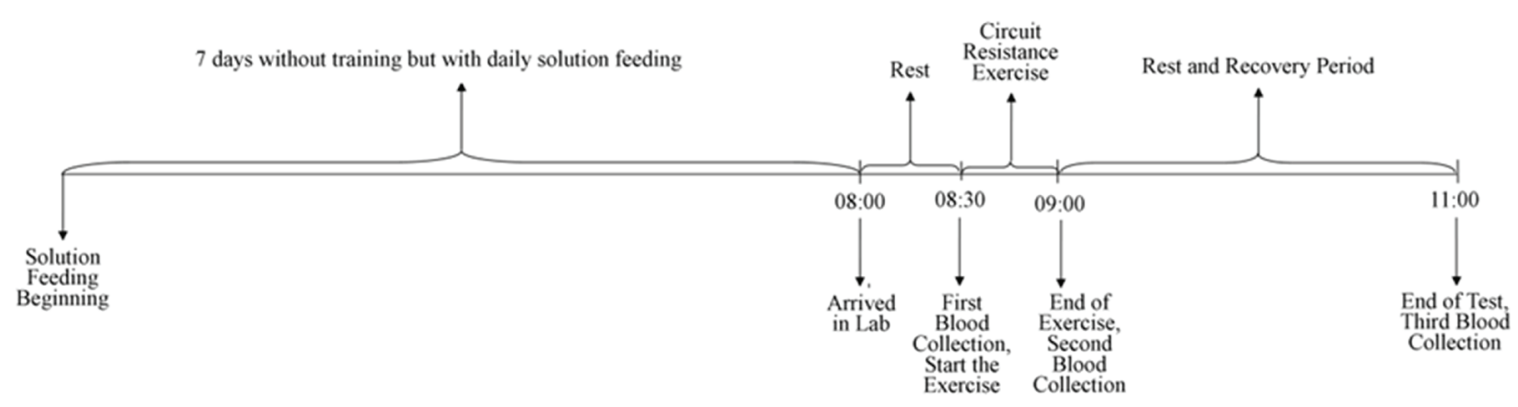

Figure 2. Research Design and Blood Collection

added into the mixture of ACD \& blood, and after adequate mixing, it settled at room temperature until separation was complete. The yellowish supernatant was separated and centrifuged. After breaking the pellet by discarding the supernatant and re-suspension in $\mathrm{dsdH}_{2} \mathrm{O}$ at $20 \mathrm{~s}$, it was mixed with $0.6 \mathrm{M} \mathrm{KCl}$ and the solution diluted with PBS. After centrifugation, the supernatant was discarded and the pellet resuspended in PBS. The cell suspension was layered over Ficoll-Hypaque and centrifuged. Finally, the supernatant was discarded, and neutrophils resuspended in HBSS. Trypan Blue Viability Test showed that cell viability was $\geq 96 \%$.

\section{Protein and $\left[\mathrm{Ca}^{2+}\right]_{i}$ Assessment}

Determination of caspase-3 (E20120710034, 0.05-10 ng/ $\mathrm{ml}$ ), calpain-1 (E20120710031, 13 -800 IU/L), calpastatin (E20120710032, 16 -1000 IU/L), Bax (E20120710035, 0.3-90 $\mathrm{ng} / \mathrm{ml}$ ), and XIAP (E20120710036, 0.05-20 ng/ml) is done by Human ELISA Kits (Glory Science Co., Ltd, China). [ $\left.\mathrm{Ca}^{2+}\right]_{\mathrm{i}}$ assessed by Atomic Absorption/ Flame Emission method and SPECTROPHOTOMETER system (Shimadzu, AA-670). Statistical Analysis
Repeated measure (two-way) ANOVA was used to determine the interaction effects of TIME and SOLUTION by SPSS software at a significance level of $\mathrm{p}<0.05$. All data were presented as means with standard deviation (Mean $\pm \mathrm{SD}$ ).

\section{Results \\ $\left[\mathrm{Ca}^{2+}\right]_{i}$}

Sphericity assumption was met (Muchly's W=0.846; $\mathrm{p}=0.397)$. The interaction effect of TIME'GROUP was significant $\left(\mathrm{F}_{2,24}=6.252 ; \mathrm{p}=0.007 ;\right.$ Partial Eta Squared $\left.=0.343\right)$ and this effect was Linear $\left(\mathrm{F}_{1,12}=8.731 ; \mathrm{p}=0.012\right.$; Partial Eta Squared $=0.421)$. Accordingly, $\left[\mathrm{Ca}^{2+}\right]_{\mathrm{i}}$. levels increased linearly in the placebo group during circuit resistance exercise and recovery period (Pre.CRE $=1.76 \pm 0.05$, Im.Po.CRE $=1.83 \pm 0.04, \quad 120 \mathrm{~m}$. Po.CRE $=1.86 \pm 0.02 \mathrm{mg} / \mathrm{L})$. In contrast, in the jujube group $\left[\mathrm{Ca}^{2+}\right]_{\mathrm{i}}$. levels remained unchanged over time (Pre.CRE $=1.91 \pm 0.07$, Im.Po. $\mathrm{CRE}=2.01 \pm 0.05,120 \mathrm{~m}$. Po.CRE $=2.14 \pm 0.02 \mathrm{mg} / \mathrm{L})[($ Fig.3-a $)]$.

\section{Calpastatin}

Sphericity assumption was met (Muchly's W=0.91; $\mathrm{p}=0.597)$. The interaction effect of TIME'GROUP was no
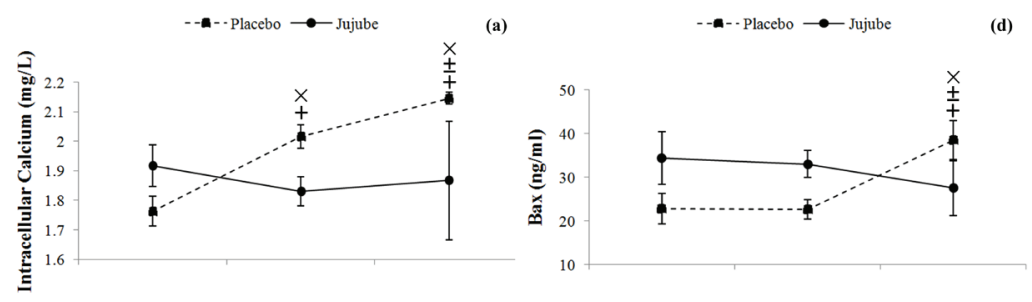
(e)
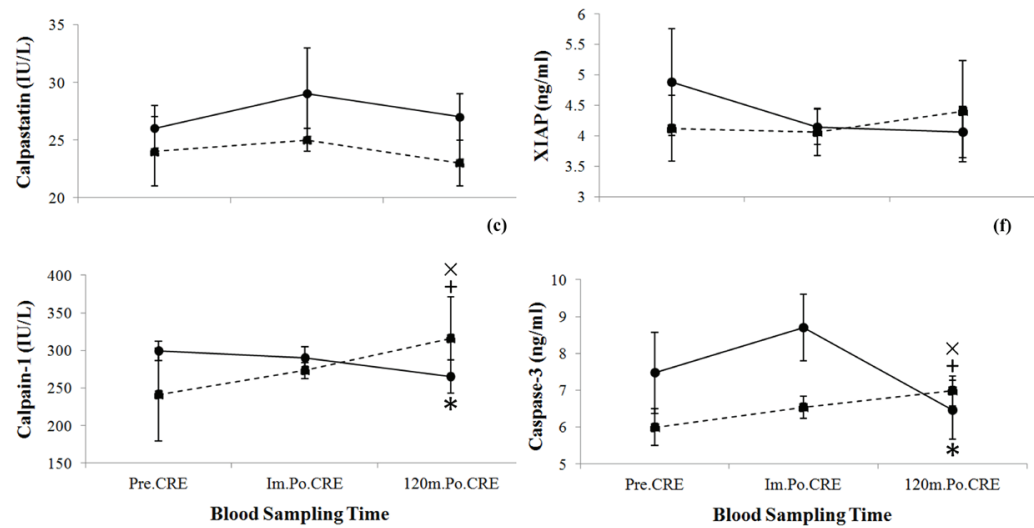

Figure 3. The Effect of One Week Supplementation with Ziziphus jujuba on some Pro- and Anti-Apoptotic Protein Levels of Human Neutrophils in Response to a Session of Circuit Resistance Exercise (a) Effect on intracellular calcium levels (b) Effect on Calpastatin expression (c) Effect on Calpain-1 expression (d) Effect on Bax expression (e) Effects on XIAP levels (f) Effects on Caspase-3 levels Pre: previous CRE: circuit resistance exercise Im.po: Immediately Post $120 \mathrm{~m}: 120$ minutes ': interaction effect of GROUP'TIME is significant at $p<0.05+$ : Significant difference with Pre.CRE in Placebo at $p<0.05 \pm$ : Significant difference with Im.Po.CRE in Placebo at $p<0.05 *$ : Significant difference with Pre.CRE in Jujube at $p<0.05$ (Error Bars present as SD) 
significant $\left(\mathrm{F}_{2,24}=2.003 ; \mathrm{p}=0.157\right.$; Partial Eta Squared $\left.=0.143\right)$. Accordingly, calpastatin levels remained unchanged after exercise in both placebo (Pre.CRE $=24 \pm 3$, Im.Po.CRE $=25 \pm 1$, $120 \mathrm{~m}$. Po.CRE $=27 \pm 2 \mathrm{IU} / \mathrm{L}$ ) as well as jujube (Pre.CRE $=26 \pm 2$, Im.Po.CRE=29 \pm 4, 120m.Po.CRE=27 $\pm 2 \mathrm{IU} / \mathrm{L}$ ) groups (Fig.3-b).

\section{Calpain-1}

Sphericity assumption was met (Muchly's W=0.619; $\mathrm{p}=0.071)$. The interaction effect of TIME'GROUP was significant $\left(\mathrm{F}_{2,24}=6.531 ; \mathrm{p}=0.005\right.$; Partial Eta Squared $\left.=0.352\right)$ and this effect was Linear $\left(\mathrm{F}_{1,12}=6.531 ; \mathrm{p}=0.016\right.$; Partial Eta Squared=0.397). Although levels remained unchanged immediately after exercise in both placebo (Pre.CRE $=241 \pm 62$, Im.Po.CRE $=273 \pm 11 \mathrm{IU} / \mathrm{L})$ as well as jujube (Pre.CRE $=299 \pm 13$, Im.Po.CRE $=290 \pm 15 \mathrm{IU} / \mathrm{L}$ ) groups, a significant elevation and placebo $(120 \mathrm{~m}$. Po.CRE $=316 \pm 55 \mathrm{IU} / \mathrm{L})$ and decline in the jujube $(120 \mathrm{~m}$. Po.CRE $=265 \pm 22 \mathrm{IU} / \mathrm{L})$ group was found at 120 after exercise (Fig.3-c).

\section{Bax}

Sphericity assumption was met (Muchly's W=0.636; $\mathrm{p}=0.083$ ). The interaction effect of TIME'GROUP was significant $\left(\mathrm{F}_{2,24}=24.509 ; \mathrm{p}<0.001\right.$; Partial Eta Squared $\left.=0.671\right)$ and this effect was both Linear $\left(\mathrm{F}_{1,12}=24.742 ; \mathrm{p}<0.001\right.$; Partial Eta Squared $=0.673)$ and Quadratic $\left(\mathrm{F}_{1,12}=23.666 ; \mathrm{p}<0.001\right.$; Partial Eta Squared=0.664)]. Accordingly, in the placebo group, there were no changes during exercise (Pre.CRE $=22.8 \pm 3.5$, Im.Po.CRE $=22.7 \pm 2.2 \mathrm{ng} / \mathrm{ml}$ ), but levels were significantly elevated during recovery period (120m.Po.CRE $=38.5 \pm 3.4 \mathrm{ng} /$ $\mathrm{ml})$. In contrast, no changes were observed at all time points in the jujube group (Pre.CRE=34.4 \pm 6.1, Im.Po.CRE $=33 \pm 3.1$, $120 \mathrm{~m}$. Po.CRE $=27.5 \pm 6.2 \mathrm{ng} / \mathrm{ml})[($ Fig.3-d) $]$.

\section{$X I A P$}

Sphericity assumption was met (Muchly's W=0.835; $\mathrm{p}=0.371)$. The interaction effect of TIME'GROUP was significant $\quad\left(\mathrm{F}_{2,24}=3.262 ; \mathrm{p}=0.056 ;\right.$ Partial Eta Squared=0.214)]. Accordingly, levels remained unchanged in both placebo (Pre.CRE $=4.12 \pm 0.54$, Im.Po. $\mathrm{CRE}=4.06 \pm 0.39,120 \mathrm{~m}$. Po.CRE $=4.40 \pm 0.83 \mathrm{ng} / \mathrm{ml})$ as well as jujube (Pre.CRE $=4.88 \pm 0.88$, Im.Po.CRE $=4.14 \pm 0.29,120 \mathrm{~m}$. Po.CRE $=4.06 \pm 0.29 \mathrm{ng} / \mathrm{ml}$ ) groups over time (Fig.3-e).

\section{Caspase-3}

Sphericity assumption was met (Muchly's W=0.959; $\mathrm{p}=0.794)$. The interaction effect of TIME'GROUP was significant $\left(\mathrm{F}_{2,24}=12.053 ; \mathrm{p}<0.0015 ;\right.$ Partial Eta Squared $\left.=0.501\right)$ and this effect was both Linear $\left(\mathrm{F}_{1,12}=14.623 ; \mathrm{p}=0.002\right.$; Partial Eta Squared=0.549) and Quadratic $\left(\mathrm{F}_{1,12}=10.189 ; \mathrm{p}=0.008\right.$; Partial Eta Squared=0.459). Although caspase-3 levels remained unchanged during exercise in both placebo (Pre. $\mathrm{CRE}=6.0 \pm 0.5$, Im.Po.CRE $=6.5 \pm 0.3 \mathrm{ng} / \mathrm{ml}$ ) as well as jujube (Pre.CRE $=7.4 \pm 1.1, \quad$ Im.Po.CRE $=8.7 \pm 0.9 \mathrm{ng} / \mathrm{ml})$ groups, concentrations significantly differ in the post-exercise period placebo (120m.Po.CRE=6.9 $\pm 0.4 \mathrm{ng} / \mathrm{ml})$, jujube $(120 \mathrm{~m}$. Po.CRE $=6.4 \pm 0.8 \mathrm{ng} / \mathrm{ml}$ ) groups (Fig.3-f).

\section{Discussion}

The results of the current study showed that neutrophils $\left[\mathrm{Ca}^{2+}\right]_{\mathrm{i}}$ levels increased linearly during and after circuit resistance exercise. The jujube supplementation attenuated the increase of $\left[\mathrm{Ca}^{2+}\right]_{\mathrm{i}}$. Although calpastatin and XIAP concentrations were unchanged in both groups and at all times, the calpain-1 levels of neutrophils increased linearly during and after exercise. However, levels of calpain-1 decreased in the jujube group and showed a similar regulation as $\left[\mathrm{Ca}^{2+}\right]_{\mathrm{i}}$ levels. During recovery, Bax and caspase- 3 increased in the placebo group, while it attenuated and decreased due to jujube supplementation. These results suggest that intensive circuit resistance exercise increases the pro-apoptotic proteins in human neutrophils, effectively suppressed by one-week supplementation with a jujube solution.

It was previously shown that intensive bouts of exercise could modulate neutrophil apoptosis. Mechanistically, this process can result either from a reduction in anti-apoptotic protein levels and/or elevation of pro-apoptotic protein levels (Kroemer et al., 1998). In neutrophils, it was reported that the cytosolic non-caspase cysteine protease calpain mediates apoptosis. Calpains are constitutively active in neutrophils but are kept in check by the endogenous inhibitor calpastatin. During neutrophil apoptosis, calpastatin is degraded, and calpains were shown to cleave Bax and to deactivate XIAP, thus causing neutrophil apoptosis (Geering \& Simon, 2011). However, the neutrophils' $\left[\mathrm{Ca}^{2+}\right]_{\mathrm{i}}$-calpastatin-calpain axis had not previously been investigated during exercise conditions. Regarding apoptosis regulation in neutrophils during exercise, it is found that resistance exercise $(90 \mathrm{~min}$, three sets, $2 \mathrm{~min}$ rest period between exercises, 3 min rest between sets) with moderate intensity $(60 \% 1 \mathrm{RM})$ had no effect on neutrophils apoptosis. As we know, calpastatin with calpain-1 are critical elements in the proximal location of the pro-apoptotic cascade, which cause the activation of Bax, mitochondria, and caspase-3, and so it seems that their expression affect the neutrophils' life span under pathologic situations (Altznauer et al., 2004). However, the intracellular protein expression of calpastatin did not change significantly in this study, but based on its changed behaviour, it could make the calpain-1 active, because the low decrease of protein levels can cause enzymatic activity.

In contrast, both intensive resistance exercise and marathon running delayed neutrophils' apoptosis during the 3 h recovery period. In parallel, it was noted that apoptosis modulation was accompanied by enhanced intracellular calcium $\left(\left[\mathrm{Ca}^{2+}\right]_{\mathrm{i}}\right)$ transients (Mooren et al., 2012). Mechanistically, it was shown that the expression of Granulocyte ColonyStimulating Factor (G-CSF) during exercise might affect the delay of neutrophils' apoptosis by inhibition of caspase-3 and -9. Similarly, it strongly inhibited the activation of calcium-dependent cysteine proteases calpains (upstream of caspase-3) through the control of calcium permeation. Calpain inhibition resulted in the stability of XIAP and thus the inhibition of caspase- 3 and - 9 activity (van Raam et al., 2008). In this study, however we saw no significant changes in XIAP, but its behaviour changes in the jujube group were in line with calpain-1 changes and confirm the findings of the literature. However, other studies found contrasting results. An increased neutrophil apoptosis after exercise was found, which was attenuated by administration of glutamine. It was suggested that glutamine exhibits protective effects on mitochondrial integrity (Lagranha et al., 2004). In another study, it is reported that one session of exercise could increase caspase- 3 gene expression in rat neutrophils by $52 \%$. In contrast, exercise with supplementation of glutamine reduced the expression of caspase- 3 by $63 \%$ in rested rats and by $75 \%$ 
in exercised rats (Lagranha et al., 2007).

Based on the modulation of neutrophil apoptosis by nutritional supplements like jujube, some ideas for possible mechanisms can be derived. Jujube consists of $77 \%$ carbohydrates , $19 \%$ fat , $4.5-5.6 \%$ protein, various amino acids, such as glycine, histidine, leucine, isoleucine, phenylalanine, proline, serine, threonine, and three precursors of glutamine, such as alanine, aspartic acid, glutamic acid (Ghanbari Niaki et al., 2013; Ghanbari Niaki et al., 2013). Accordingly, it is suggested that glutamine and glucose might affect jujube effects on neutrophil apoptosis modulation during exercise. Both contents are the main energy sources of neutrophils (Lagranha et al., 2008). Generally, neutrophils are cells that use glutamine intensively for metabolism (Curi et al., 1997). Accordingly, jujube supplementation for one week may provide additional energy sources for neutrophils, which helps to stabilize cell metabolism during exercise conditions. In this regard, it is reported that 30 days of administration with jujube extraction reduced Bax expression and increased Bcl-2 expression in rats' heart muscle in response to two 15-min swimming bouts on two different days (Liang \& Juan, 2011).

Besides providing energy, jujube fruit contains various minerals and vitamins, which might affect neutrophils' oxidative statuses; these include iron (involved in catalase), zinc (involved in superoxide dismutase), manganese (involved in superoxide dismutase), and vitamins $\mathrm{C}$ and $\mathrm{A}$. In addition, also other jujube contents, such as glycoside complexes, including phenols (Quercetin and Kaemferol) as well as flavonoids and triterpenes, are known to have anti-oxidative properties (Afroz et al., 2014; Cheng et al., 2012; Choi et al, 2011; Gao et al., 2011; Gao et al., 2012). During exercise, it is known that increased oxygen turnover, tissue damage, and ischemia-reperfusion processes are followed by increased production of reactive oxygen species (ROS) (Afzalpour, et al., 2015; Afzalpour et al., 2014). In this regard, it was shown that repeated high-intensity acute exercise increased apoptosis in neutrophils (Syu et al., 2011; Tayebi et al., 2014), which were related to an increased oxidative state (Syu et al., 2011). Three weeks of jujube administration $(0.4 \mathrm{~g} / \mathrm{kg}$ body weight) reduced apoptosis and affected total antioxidant capacity (TAC) (Afzalpour et al., 2014) and glutathione peroxidase (GPX) levels (Afzalpour et al., 2015). It is found that 30 days of administration with jujube extraction reduced Bax expression and increased $\mathrm{Bcl}-2$ expression in rats' heart muscle. It is assumed that the extraction reduced levels of lipid peroxidation and increased antioxidant enzyme activities in the heart muscle (Liang \& Juan, 2011). Accordingly, the antioxidant effect of jujube might be an additional primary mechanism for reducing pro-apoptotic protein concentrations in neutrophils in response to an intensive circuit resistance exercise.

In summary, the present data indicate that a single session of circuit resistance exercise increased the levels of pro-apoptotic proteins (calpain-1, Bax, caspase-3) in human neutrophils. It is assumed that the $\left[\mathrm{Ca}^{2+}\right]_{\mathrm{i}}$-Calpastatin-Calpai mechanistically $\mathrm{n}$ axis upstream affects caspase-3. Supplementation with a jujube solution effectively suppressed these effects and might inhibit neutrophils' apoptosis. It is supposed that jujube might be an additional energy source for neutrophils or might protect neutrophils from the detrimental effects of ROS. However, clinical relevance for jujube supplementation during exercise has to be proved in future studies.

\section{References}

Afroz, R., Tanvir, E. M., Islam, M. A., Alam, F., Gan, S. H., \& Khalil, M. I. (2014). Potential Antioxidant and Antibacterial Properties of a Popular Jujube Fruit: Apple Kul (Zizyphus mauritiana). Journal of Food Biochemistry, 38(6), 592-601. doi: 10.1111/jfbc. 12100

Afzalpour, M. E., Abtahi Eivari, H., Rezazadeh, A., \& Soluki, A. (2015). Effect of Ziziphus jujuba Supplementation before One Session of Acute Resistance Exercise on the Serum Glutathione Peroxidase and Superoxide Dismutase Activity. Horizon of Medical Sciences, 21(2), 97-104.

Afzalpour, M. E., Rezazadeh, A., \& Abtahi, S. H. (2014). Effects of Jujube fruit on total antioxidant capacity and lipid peroxidation in young women after an intensive resistance exercise session. Journal of Sport and Biomotor Sciences, 6(11), 16-26.

Altznauer, F., Conus, S., Cavalli, A., Folkers, G., \& Simon, H. U. (2004). Calpain-1 regulates Bax and subsequent Smacdependent caspase-3 activation in neutrophil apoptosis. Journal of Biological Chemistry, 279(7), 5947-5957. doi: 10.1074/jbc.M308576200

Borges, L., Passos, M. E. P., Silva, M. B. B., Santos, V. C., Momesso, C. M., Pithon-Curi, T. C., . . . Hatanaka, E. (2019). Dance Training Improves Cytokine Secretion and Viability of Neutrophils in Diabetic Patients. Mediators of Inflammation, 2019, 2924818. doi: 10.1155/2019/2924818

Chen, J., Li, Z., Maiwulanjiang, M., Zhang, W. L., Zhan, J. Y. X., Lam, C. T. W., . . . Tsim, K. W. K. (2013). Chemical and Biological Assessment of Ziziphus jujuba Fruits from China: Different Geographical Sources and Developmental Stages. Journal of Agricultural and Food Chemistry, 61(30), 7315-7324. doi: 10.1021/jf402379u

Chen, Y.-C., Chou, W.-Y., Fu, T.-C., \& Wang, J.-S. (2018). Effects of normoxic and hypoxic exercise training on the bactericidal capacity and subsequent apoptosis of neutrophils in sedentary men. European Journal of Applied Physiology, 118(9), 1985-1995. doi: 10.1007/s00421-0183935-7

Cheng, D., Zhu, C., Cao, J., \& Jiang, W. (2012). The protective effects of polyphenols from jujube peel (Ziziphus Jujube Mill) on isoproterenol-induced myocardial ischemia and aluminum-induced oxidative damage in rats. Food and Chemical Toxicology, 50(5), 1302-1308. doi: 10.1016/j. fct.2012.01.026

Choi, S. H., Ahn, J. B., Kozukue, N., Levin, C. E., \& Friedman, M. (2011). Distribution of free amino acids, flavonoids, total phenolics, and anti-oxidative activities of Jujube (Ziziphus jujuba) fruits and seeds harvested from plants grown in Korea. Journal of Agricultural and Food Chemistry, 59(12), 6594-6604. doi: 10.1021/jf200371r

Chuong, P., Wysoczynski, M., \& Hellmann, J. (2019). Do Changes in Innate Immunity Underlie the Cardiovascular Benefits of Exercise? Frontiers in Cardiovascular Medicine, 6(70). doi: 10.3389/fcvm.2019.00070

Curi, T. C., De Melo, M. P., De Azevedo, R. B., Zorn, T. M., \& Curi, R. (1997). Glutamine utilization by rat neutrophils: presence of phosphate-dependent glutaminase. American Journal of Physiology, 273(4 Pt 1), C1124-1129.

Cury-Boaventura, M. F., Levada-Pires, A. C., Folador, A., Gorjao, R., Alba-Loureiro, T. C., Hirabara, S. M., . . . Pithon-Curi, T. C. (2008). Effects of exercise on leukocyte death: prevention by hydrolyzed whey protein enriched 
with glutamine dipeptide. European Journal of Applied Physiology, 103(3), 289-294. doi: 10.1007/s00421-0080702-1

Du, L.-J., Gao, Q.-H., Ji, X.-L., Ma, Y.-J., Xu, F.-Y., \& Wang, M. (2013). Comparison of Flavonoids, Phenolic Acids, and Antioxidant Activity of Explosion-Puffed and Sun-Dried Jujubes (Ziziphus jujuba Mill.). Journal of Agricultural and Food Chemistry, 61(48), 11840-11847. doi: 10.1021/ jf401744c

Ermert, D., Niemiec, M. J., Röhm, M., Glenthøj, A., Borregaard, N., \& Urban, C. F. (2013). Candida albicans escapes from mouse neutrophils. Journal of Leukocyte Biology, 94(2), 223-236. doi: 10.1189/jlb.0213063

Gao, Q. H., Wu, C. S., Yu, J. G., Wang, M., Ma, Y. J., \& Li, C. L. (2012). Textural characteristic, antioxidant activity, sugar, organic acid, and phenolic profiles of 10 promising jujube (Ziziphus jujuba Mill.) selections. Journal of Food Science, 77(11), C1218-C1225.

Gao, Q.-H., Wu, P.-T., Liu, J.-R., Wu, C.-S., Parry, J. W., \& Wang, M. (2011). Physico-chemical properties and antioxidant capacity of different jujube (Ziziphus jujuba Mill.) cultivars grown in loess plateau of China. Scientia Horticulturae, 130(1), 67-72. doi: 10.1016/j.scienta.2011.06.005

Geering, B., \& Simon, H. U. (2011). Peculiarities of cell death mechanisms in neutrophils. Cell Death \& Differentiation, 18(9), 1457-1469.

Ghanbari Niaki, A., Hosseini, F., Rooadbari, F., Rahmati Ahmadabad, S., \& Rooadbari, M. (2013). Effects of Aerobic Training, with or without Zizyphus Jujuba Water Extraction, on Fundus Nesfatin-1, ATP, HDL-C, and LDL-C Concentrations in Female Rats. Iranian Journal of Health and Physical Activity, 4(1), 9-16.

Ghanbari Niaki, A., Mohammadi Joojadeh, F., Zare Kookandeh, N., Najafi, S., Chaichi, M. J., Rodbari, F., \& Bayat, H. (2013). Liver and plasma nesfatin-1 responses to 6 weeks of treadmill running with or without zizyphus jujuba liquid extract in female rat. International Journal of Endocrinology and Metabolism, 11(2), 95-101. doi: $10.5812 / \mathrm{ijem} .8438$

Guo, S., Duan, J.-a., Qian, D., Tang, Y., Qian, Y., Wu, D., . . Shang, E. (2013). Rapid Determination of Amino Acids in Fruits of Ziziphus jujubaby Hydrophilic Interaction UltraHigh-Performance Liquid Chromatography Coupled with Triple-Quadrupole Mass Spectrometry. Journal of Agricultural and Food Chemistry, 61(11), 2709-2719. doi: 10.1021/jf305497r

Guo, S., Duan, J.-a., Qian, D., Wang, H., Tang, Y., Qian, Y., . . . Shang, E. (2013). Hydrophilic interaction ultra-high performance liquid chromatography coupled with triple quadrupole mass spectrometry for determination of nucleotides, nucleosides and nucleobases in Ziziphus plants. Journal of Chromatography A, 1301, 147-155. doi: 10.1016/j.chroma.2013.05.074

Jacobs, L., Nawrot, T. S., de Geus, B., Meeusen, R., Degraeuwe, B., Bernard, A., ... Panis, L. I. (2010). Subclinical responses in healthy cyclists briefly exposed to traffic-related air pollution: an intervention study. Environmental Health, 9(1), 64. doi: 10.1186/1476-069x-9-64

Kroemer, G., Dallaporta, B., \& Resche-Rigon, M. (1998). The mitochondrial death/life regulator in apoptosis and necrosis. Annual Review of Physiology, 60, 619-642. doi: 10.1146/annurev.physiol.60.1.619
Kruger, K., \& Mooren, F. C. (2014). Exercise-induced leukocyte apoptosis. Exercise Immunology Review, 20, 117-134.

Lagranha, C. J., deLima, T. M., Senna, S. M., Doi, S. Q., Curi, R., \& Pithon-Curi, T. C. (2005). The effect of glutamine supplementation on the function of neutrophils from exercised rats. Cell Biochemistry \& Function, 23(2), 101107. doi: $10.1002 /$ cbf. 1192

Lagranha, C. J., Hirabara, S. M., Curi, R., \& Pithon-Curi, T. C. (2007). Glutamine supplementation prevents exerciseinduced neutrophil apoptosis and reduces p38 MAPK and JNK phosphorylation and p53 and caspase 3 expression. Cell Biochemistry \& Function, 25(5), 563-569. doi: 10.1002/ cbf. 1421

Lagranha, C. J., Levada-Pires, A. C., Sellitti, D. F., Procopio, J., Curi, R., \& Pithon-Curi, T. C. (2008). The effect of glutamine supplementation and physical exercise on neutrophil function. Amino Acids, 34(3), 337-346. doi: 10.1007/s00726-007-0560-x

Lagranha, C. J., Senna, S. M., De Lima, T. M., Silva, É. P. P., Doi, S. Q., Curi, R. U. I., \& Pithon-Curi, T. C. (2004). Beneficial Effect of Glutamine on Exercise-Induced Apoptosis of Rat Neutrophils. Medicine \& Science in Sports \& Exercise, 36(2), 210-217.

Lee, S. M., Min, B. S., Lee, C. G., Kim, K. S., \& Kho, Y. H. (2003). Cytotoxic Triterpenoids from the Fruits of Zizyphus jujuba. Planta Medica, 69(11), 1051-1054. doi: 10.1055/s-2003-45155

Li, J., Ai, L., Yang, Q., Liu, Y., \& Shan, L. (2013). Isolation and structural characterization of a polysaccharide from fruits of Zizyphus jujuba cv. Junzao. International Journal of Biological Macromolecules, 55, 83-87. doi: 10.1016/j. ijbiomac.2012.12.017

Liang, S., \& Juan, J. (2011). Effect of jujube extract on oxidative injury in heart muscles of exhausted training rats. African Journal of Microbiology Research, 5(14), 1896-1899.

Mooren, F. C., Völker, K., Klocke, R., Nikol, S., Waltenberger, J., \& Krüger, K. (2012). Exercise delays neutrophil apoptosis by a G-CSF-dependent mechanism. Journal of Applied Physiology, 113(7), 1082-1090. doi: 10.1152/ japplphysiol.00797.2012

Plastina, P. (2016). Pharmacological Aspects of Jujubes. Pharmacologia, 7(5), 243-255. doi: 10.5567/ pharmacologia.2016.243.255

Presbitero, A., Mancini, E., Castiglione, F., Krzhizhanovskaya, V. V., \& Quax, R. (2019). Game of neutrophils: modeling the balance between apoptosis and necrosis. BMC Bioinformatics, 20(6), 475. doi: 10.1186/s12859-019-30446

Rodríguez Villanueva, J., \& Rodríguez Villanueva, L. (2017). Experimental and Clinical Pharmacology of Ziziphus jujuba Mills. Phytotherapy Research, n/a-n/a. doi: 10.1002/ ptr.5759

Syu, G. D., Chen, H. I., \& Jen, C. J. (2011). Severe Exercise and Exercise Training Exert Opposite Effects on Human Neutrophil Apoptosis via Altering the Redox Status. PLoS One, 6(9), e24385. doi: 10.1371/journal.pone.0024385

Tayebi, S. M., Agha-Alinejad, H., Shafaee, S., Gharakhanlou, R., \& Asouri, M. (2014). Short-Term Effects of Oral Feeding Jujube Ziziphus Solution before a Single Session of Circuit Resistance Exercise on Apoptosis of Human Neutrophil. Annals of Applied Sport Science, 2(1), 53-68.

Tayebi, S. M., Ghorban-alizadeh, F., Ghaziani., \& Mohamadi, 
H., Bazneshin. (2012). Oral glucose administration after circuit resistance exercise induced plasma ghrelin suppression. European Journal of Experimental Biology, 2(5), 1616-1624.

Tayebi, S. M., Saeidi, A., Gharahcholo, L., Haghshenas Gatabi, R., \& Radmehr, L. (2016). Acute and Short-Term Effects of Oral Jujube Solution on White Blood Cell and its Differential count in Response to Circuit Resistance Exercise. International Journal of Applied Exercise Physiology, 5(2), 1-10.

Tayebi, S. M., Saeidi, A., Mahmoudi, A. A., Gharahcholo, L., \& Radmehr, L. (2015). Acute and Short-Term Effects of Oral Feeding of Jujube Solution on Blood Platelets and its Morphological Indices in Response to a Circuit Resistance Exercise. Annals of Applied Sport Science, 3(3), 67-82. doi: 10.18869/acadpub.aassjournal.3.3.67
Vahedi, F., Fathi Najafi, M., \& Bozari, K. (2008). Evaluation of inhibitory effect and apoptosis induction of Zyzyphus Jujube on tumor cell lines, an in vitro preliminary study. Cytotechnology, 56(2), 105-111. doi: 10.1007/s10616-0089131-6

van Raam, B. J., Drewniak, A., Groenewold, V., van den Berg, T. K., \& Kuijpers, T. W. (2008). Granulocyte colonystimulating factor delays neutrophil apoptosis by inhibition of calpains upstream of caspase-3. Blood, 112(5), 20462054. doi: 10.1182/blood-2008-04-149575

Wang, B.-N., Liu, H. F., Zheng, J. B., Fan, M. T., \& Cao, W. (2011). Distribution of Phenolic Acids in Different Tissues of Jujube and Their Antioxidant Activity. Journal of Agricultural and Food Chemistry, 59(4), 1288-1292. doi: $10.1021 /$ jf103982q 